\title{
Experimental evaluation of the impact of carbon nanotube EUV pellicles on reticle imaging
}

\author{
lacopo Mochi, ${ }^{\mathrm{a}, \star}$ Marina Y. Timmermans, ${ }^{\mathrm{b}}$ Emily E. Gallagher, ${ }^{\mathrm{b}}$ Marina Mariano, ${ }^{\mathrm{b}}$ Ivan Pollentier, ${ }^{\mathrm{b}}$ Rajeev Rajendran, ${ }^{\mathrm{a}}$ \\ Patrick Helfenstein, ${ }^{\mathrm{a}}$ Sara Fernandez, ${ }^{\mathrm{a}}$ Dimitrios Kazazis, ${ }^{\mathrm{a}}$ and Yasin Ekinci ${ }^{\mathrm{a}}$ \\ ${ }^{a}$ Paul Scherrer Institut, Villigen, Switzerland \\ 'IMEC, Leuven, Belgium
}

\begin{abstract}
Background: The purpose of EUV pellicles is to protect the surface of EUV lithography masks from particle contamination. It is important to ensure that the optical characteristics of the pellicle membrane do not critically affect the reticle image quality.

Aim: We want to verify the possibility to integrate pellicle inspection and characterization capabilities in reflective-mode EUV mask scanning microscope (RESCAN), our actinic mask inspection platform based on coherent diffraction imaging.

Approach: We studied the impact of a few selected EUV pellicle prototypes on the quality and the contrast of the reticle image obtained with RESCAN.

Results: We measured the scattering distribution of the pellicles, and we correlated it with the mask image contrast and fidelity. We also detected the presence of a $6.5-\mu \mathrm{m}$-diameter fiber on the pellicle surface.

Conclusions: We demonstrated that RESCAN is suitable for through-pellicle actinic mask inspection and can be also used to characterize and monitor the pellicle quality.

(C) 2019 Society of Photo-Optical Instrumentation Engineers (SPIE) [DOI: 10.1117/1.JMM.18.1.014002]

Keywords: extreme ultraviolet; pellicle; actinic pattern inspection; defect inspection; lensless microscopy; coherent diffraction imaging; carbon nanotubes.

Paper 18147 received Dec. 5, 2018; accepted for publication Feb. 26, 2019; published online Mar. 13, 2019.
\end{abstract}

\section{Introduction}

Extreme ultraviolet (EUV) masks will eventually be equipped with protective pellicles. The pellicle, installed at a distance of about $2.5 \mathrm{~mm}$ from the mask surface, will hold fall-on particles out of the imaging plane, thereby minimizing their impact on wafer yield. Although nonpellicle lithography is being considered, this is a risky and nonideal solution. The challenge is to find a membrane with suitable optical and mechanical characteristics: high transmission at EUV, low reflectivity, low scattering, low thermal expansion coefficient, and high mechanical stability. The pellicle characteristics have an impact on the imaging performance of an exposure or inspection system, and for this reason, an accurate evaluation of pellicle's optical properties must be performed. We tested different pellicle samples on reflective-mode EUV mask scanning microscope (RESCAN) to verify their impact on the aerial image formation. RESCAN is an actinic pattern inspection platform currently under development at Paul Scherrer Institute (PSI). ${ }^{1,2}$ It consists of a lensless scanning microscope based on coherent diffraction imaging $(\mathrm{CDI}){ }^{3}$ In RESCAN, the sample is illuminated with a coherent EUV beam and the reflected diffraction beam is recorded by a pixel detector. Multiple diffraction patterns are combined together to reconstruct the sample image and phase using ptychography. ${ }^{4}$ Since RESCAN does not have any imaging optics close to the reticle, there is no physical obstacle to mounting a pellicle on top of the sample, and this makes it a flexible and effective tool for studying the performance of new membranes and materials. In this work, we present the results of the image reconstruction of an EUV reticle test sample with different carbon nanotubes (CNT) pellicles with and without coating, and we compare the images to a reference obtained with the bare sample. We also present an estimate of the scattering introduced by different types of EUV-transparent membranes and its effect on the image formation. Finally, we demonstrate how RESCAN can be used not only as an actinic mask inspection platform but also as an efficient tool to inspect the pellicle surface for defects.

\section{Measurement Setup}

\subsection{RESCAN}

The measurements were carried out using RESCAN, a lensless microscope dedicated to EUV mask defect inspection operating at the XIL-II beamline of the Swiss Light Source. RESCAN's working principle consists of collecting images of the sample and comparing them to the image of a reference die or to an aerial image calculated from the reticle's design to identify the presence of defects. ${ }^{1}$ The images of the sample are obtained through CDI, which allows for retrieval of both the phase and the magnitude of the reticle's surface. The CDI approach used in RESCAN is based on ptychography and consists of scanning the sample with an EUV illumination probe, collecting the relative diffraction 
patterns and propagating them back to the sample plane with an iterative procedure to retrieve the phase and magnitude of the reticle. The sample scan is performed moving the sample along $x$ and $y$ axes with a piezomotor with about 5-nm accuracy. RESCAN is a research prototype, it is currently located in a small vacuum chamber that limits the size of the samples to $20 \times 20 \mathrm{~mm}^{2}$ and is equipped with a stage with a travel range of $200 \mu \mathrm{m}$. The optical layout of RESCAN, shown in Fig. 1(a), consists of an aperture, a focusing toroidal mirror, and a folding mirror. The XIL-II beamline is equipped with a monochromator that allows RESCAN to operate with a narrow bandwidth $\lambda / \Delta \lambda=1500$, which is an essential requirement for CDI systems. ${ }^{5}$ The beam is focused by the toroidal mirror and folded onto the reticle sample with a variable numerical aperture (NA) ranging from 0.002 to 0.015 . The beam footprint on the sample can be tuned by changing the position of the folding mirror or the focusing mirror. The beam, reflected and diffracted by the sample, is collected by a Princeton Instruments $2048 \times 2048$-pixel CCD at a distance $d$ of $62 \mathrm{~mm}$. The maximum resolution of RESCAN depends on the NA of the detector defined as

$\mathrm{NA}=\sin [\arctan (s / d)]$

where $s$ is the detector half size. The detector used for the measurements presented here has a pixel size of $13.5 \mu \mathrm{m}$ and its half size, $s$, is $13.8 \mathrm{~mm}$. The image resolution is, therefore,

$r \simeq \frac{\lambda}{2 \mathrm{NA}}=35 \mathrm{~nm}$

RESCAN is equipped with the adjustable pellicle mount sketched in Fig. 1(b). It consists of a $35 \times 35 \mathrm{~mm}^{2}$ support that can be installed as close as $0.5 \mathrm{~mm}$ from the sample surface and can hold a pellicle sample mounted on a $30 \times 30 \mathrm{~mm}^{2}$ wafer.

\subsection{Pellicle Samples}

The development of suitable EUV pellicles is currently ongoing. Various types of pellicle membranes have been evaluated and tested both experimentally and with simulations to assess their mechanical and optical characteristics. ${ }^{6-9}$ Among them, CNT pellicles proved to be a promising option with high EUV transmission (up to $\sim 99 \%$ ), low reflectivity $0.001 \%$, and good mechanical stability $(<0.08 \mathrm{~mm}$ deflection under a $2-\mathrm{Pa}$ pressure for a $10 \times 10 \mathrm{~mm}^{2}$ sample). ${ }^{6,10}$
The lifetime of CNT membranes can be limited by the exposure to hydrogen ions generated by EUV radiation inside the scanner. To mitigate this effect, it is possible to coat the membrane with a protective layer. The selection of the coating material is dictated by the EUV pellicle requirements: optical properties ( $n$ and $k$ ) of the coating must allow the full membrane solution to achieve EUV transmission $>90 \%$, low reflection and scattering. The coating material must also be compatible with EUV exposure of $300 \mathrm{~W}$ and greater power in the presence of hydrogen. ${ }^{7}$ Currently, the development of suitable coatings deposited by a technique compatible with the free-standing CNT membrane is in progress but is not the focus of the current publication. In this study, we examined different CNT-based pellicles including random single-walled (SW) CNTs [Fig. 2(a)] with different protective metal coatings and aligned multiwalled (MW) CNTs [Fig. 2(b)] with and without support mesh.

The EUV transmission of SWCNT-based pellicle, used in this study, without the coating is $97.6 \%$ and with the coating it is about $93 \%$, depending on the coating material and thickness, as measured at the German National Lab, PhysikalischTechnische Bundesanstalt (PTB). The EUV transmission of MWCNT-based pellicle consisting of one layer of MWCNTs is $93.4 \%$, and when supported by the macro-CNT bundles the EUV transmission is about $90 \%$.

The core material of the SWCNT-based pellicle is a network of thin, randomly oriented, SWCNT bundles synthesized using a floating catalyst chemical vapor deposition technique. ${ }^{7}$ The MWCNT-based pellicle is composed of aligned MWCNTs and bundles drawn from vertically oriented CNT arrays grown on a substrate. ${ }^{7}$ In this work, the MWCNT-based pellicles were supported with a sparse net of macro-CNT bundles with a diameter of about $7 \mu \mathrm{m}$. These bundles were explored as a method to strengthen the membrane but are no longer EUV pellicle candidates. However, the larger strands enabled the demonstration of RESCAN's pellicle inspection capabilities. All the pellicle samples shown here are based on last year's technology. More advanced prototypes of EUV pellicle membranes have now been developed and are ready to be tested.

\section{Through-Pellicle Imaging of EUV Reflective Samples}

The analysis of RESCAN raw data allows us to study the pellicle, characterize its scattering properties, and verify the presence of defects on its surface. Moreover, from the reconstructed

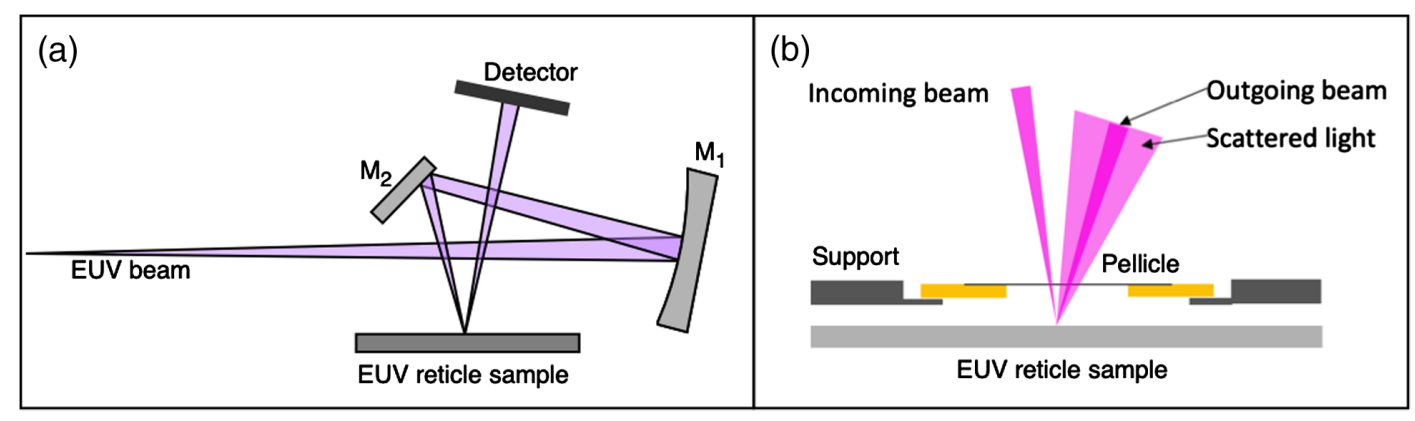

Fig. 1 (a) RESCAN optical layout. The EUV beam from the synchrotron is focused and folded onto the sample plane by a toroidal mirror (M1) and by a plane mirror (M2). Both mirrors are coated with a multilayer to ensure maximum EUV reflectivity at their respective incidence angle. The reflected and diffracted beam is collected by a CCD camera at a distance of $62 \mathrm{~mm}$ from the sample. (b) RESCAN pellicle mount detail. 
(a)

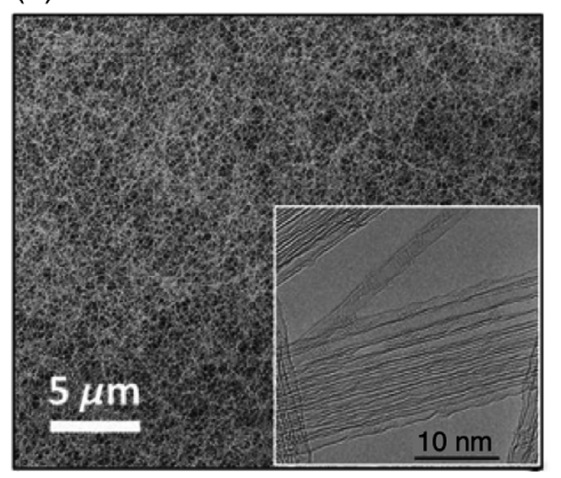

(b)

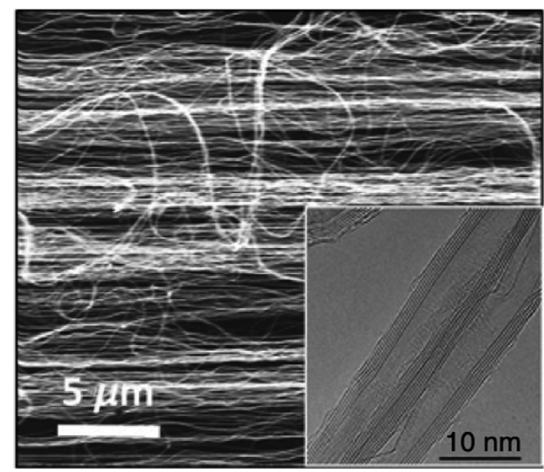

Fig. 2 Scanning electron micrographs of (a) random CNT membrane consisting of SW CNTs as shown with transmission electron micrograph at the inset and (b) aligned CNT membrane consisting of MW CNTs as shown with transmission electron micrograph at the inset. ${ }^{6}$

sample images, we can estimate the impact of the pellicle on the imaging contrast and on the pattern fidelity. The EUV mask sample used for this work is a $20 \times 20 \mathrm{~mm}^{2}$ square silicon wafer with a multilayer coating designed for maximum reflectivity $(>60 \%)$ at $13.5 \mathrm{~nm}$ and $6 \mathrm{deg}$ incidence angle. The sample is patterned with a random, logic-like design on a 140-nm layer of hydrogen silsesquioxane (HSQ). We note that this sample has lower contrast than the state-ofthe-art EUV reticles.

\subsection{Pellicle Defect Inspection}

The size of pellicle defects that can cause a significant CD error in the aerial image is on the order of magnitude of a few micrometers. ${ }^{11,12}$ The raw data collected in RESCAN consist of a set of far-field diffraction patterns obtained by illuminating the sample through the pellicle and scanning the EUV reticle. A defect on the pellicle can cast a visible shadow on the diffraction pattern as it propagates through the membrane from the sample to the detector. Figure 3 shows how a CNT support mesh is visible in the recorded diffraction pattern. Calculating the pixel magnification as the ratio between the distance of the detector and the pellicle from the focal

(a)

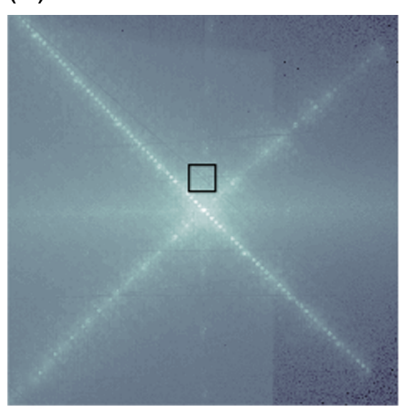

(b)

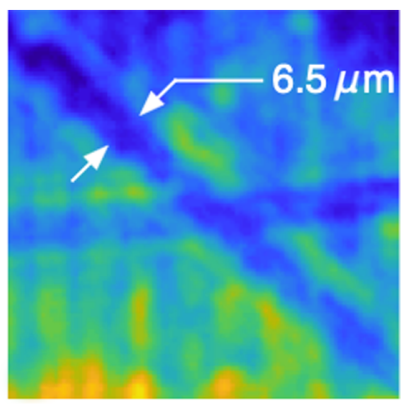

Fig. 3 (a) Diffraction pattern of a reticle sample covered by an MW-CNT pellicle with support mesh. The picture is cropped to $1800 \times$ 1800 pixels and displayed on a logarithmic intensity scale. The diffraction pattern corresponds to a Manhattan-geometry reticle with a 45 deg orientation with respect to the CCD axes. The black square delimits a region of the diffraction pattern where two crossing strands of the support mesh cast a visible shadow. (b) Detail of the diffraction pattern delimited by the black square on (a) showing the shadow of the two crossing strands. The image is displayed on a compressed logarithmic scale. plane (located on the sample) and considering the detector pixel size, we estimated the size of the CNT bundle to be $6.5 \mu \mathrm{m}$, in good agreement with the SEM measured value of $7 \mu \mathrm{m}$.

\subsection{Pellicle Scattering}

In the absence of the pellicle, the diffraction patterns recorded by RESCAN provide an immediate measure of the scattering irradiance coming from the sample surface and from the condenser optics. The pellicle generates two additional diffraction contributions from the interaction with the incoming and outgoing beam. In addition, large defects on the pellicle surface can cast a shadow on the diffraction pattern generated by the sample. In RESCAN, it is relatively straightforward to evaluate these contributions by comparing the diffraction patterns recorded through pellicle to a reference obtained from the bare sample. We inspected a region free of absorber patterns on the EUV mask sample to avoid the strong diffraction signature from the reticle pattern and simplify the data interpretation. The images in the top row of Fig. 4 show the recorded diffraction patterns in log scale. The irradiance intensity is normalized to the maximum irradiance of the no-pellicle case [Fig. 4(a)]. The images in the bottom row of Fig. 4 show the irradiance intensity of the reflected beam that corresponds to the condenser pupil projection on the detector plane. From a visual observation of the diffraction patterns, there seems to be little or no difference between the random SW-CNT pellicle [Fig. 4(b)] and the reference [Fig. 4(a)] indicating that the main effect of this membrane is the beam attenuation, visible from the comparison between the average intensities of the reflected beam. The aligned MW-CNT pellicle [Figs. 4(c) and 4(d)] shows instead a visible diffraction contribution that corresponds to the dominant scattering direction of the aligned CNTs. Although the individual CNTs are not visible in the diffraction patterns, it is possible to distinguish their alignment direction in the pupil projection image from the presence of the stripes due to the nonuniformity of the CNTs' distribution. To make a more quantitative evaluation of the pellicle scattering contribution, it is sufficient to extract the irradiance intensity from the diffraction patterns along a specific axis. However, when comparing different samples, it is convenient to use an estimator that does not depend on the scattering direction. In this case, we report the average scattering intensity as a function of the 
(a)

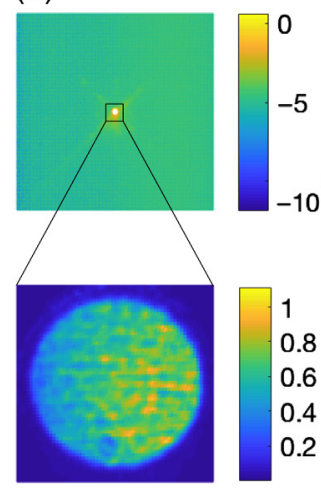

(b)
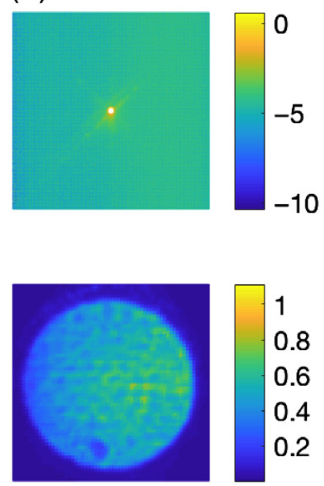

(c)
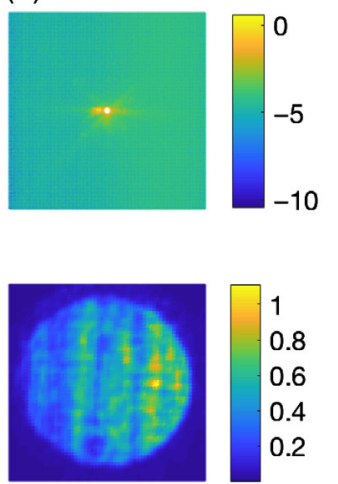

(d)
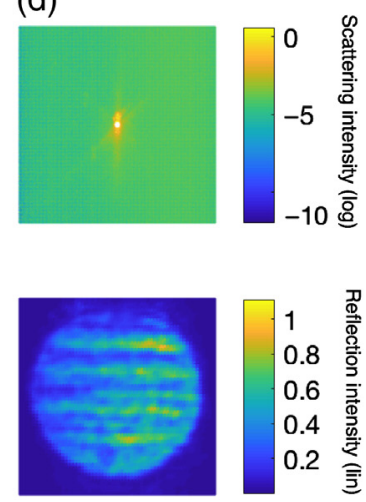

Fig. 4 The top row shows the log scale diffraction patterns observed in RESCAN. The bottom row shows the details of the zero-order diffraction or simply the projection of the exit pupil of the condenser optics displayed on a linear scale. (a) Reference case without pellicle. The image was collected on a flat region of the sample, covered with HSQ. The roughness in the pupil projection intensity and the large blemish in the lower part of the image come from the condenser mirrors surfaces. (b) Random SW CNT pellicle with metal coating. (c) Aligned MW-CNT pellicle with a support mesh (uncoated). (d) Same pellicle shown in (c) rotated by 90 deg.

(a)

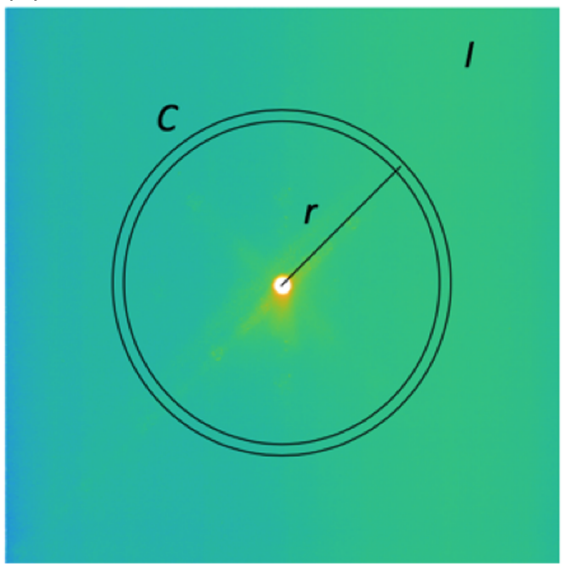

(b)

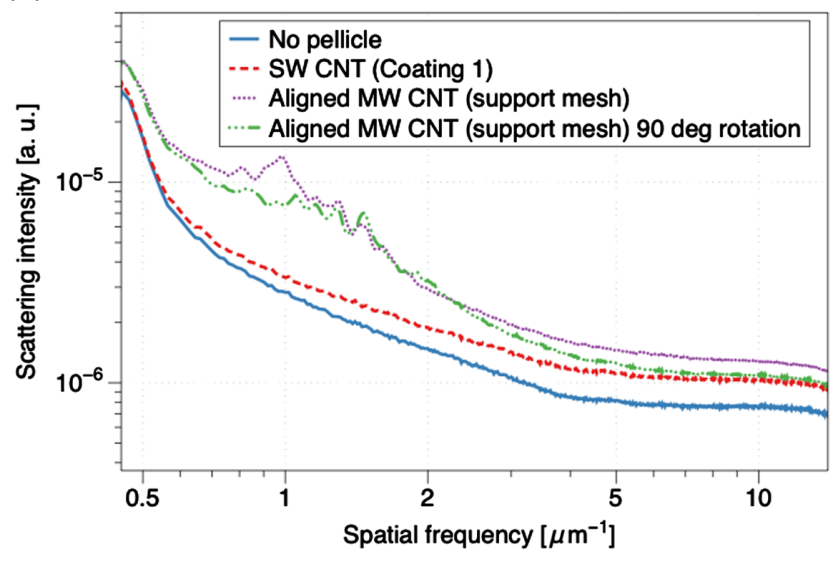

Fig. 5 (a) Evaluation of the scattering intensity as a function of the scattering angle is carried out by averaging the recorded irradiance $I$ over a circular crown $C$ centered in the middle of the entrance pupil projection. The radius $r$ of the circular crown corresponds to the scattering angle. (b) Scattering intensity as a function of the spatial frequency of the scattering features for different CNT pellicles.

diffraction. This is done by averaging the intensity over a circular crown centered in the middle of the pupil projection as shown in Fig. 5. The scattering intensity $S(r)$ is then:

$$
S(r)=\frac{1}{\pi\left(2 r \Delta r+\Delta r^{2}\right)} \int_{C} I \mathrm{~d} C,
$$

where $I$ is the measured diffraction irradiance and $r$ and $\Delta r$ are the inner radius and thickness of the circular crown $C$, respectively. Expressing $r$ as a function of the spatial frequency of the scattering features, we obtain:

$f(r)=\frac{r}{\lambda z}$,

where $\lambda$ is the wavelength and $z$ is the distance between the sample and the detector. In Fig. 5, we show the average scattering intensity as a function of the spatial frequency of the diffracting features for the pellicles shown in Fig. 4. While the scattering from the random SW-CNT pellicle with coating has the same behavior as the no-pellicle reference case, the aligned uncoated MW-CNT pellicle with a support mesh shows a higher scattering intensity in the spatial frequency range between 0.6 and $2 \mu \mathrm{m}^{-1}$. This scattering will be superimposed on the diffraction of features with the same spatial frequency range and reduce their contrast in the aerial image.

\subsection{Image Quality Evaluation}

Mask defect detection in RESCAN requires the reconstruction of the reticle image. Although this image does not necessarily correspond to the aerial image generated by a scanner where the illumination is engineered to optimize the process window, we can still use it to estimate the impact of EUV pellicles on the contrast and on the pattern fidelity. We performed through-pellicle inspections of the same sample using a constant parameter set, including illumination NA, scanning area, scanning step size, and image reconstruction algorithm. As for the scattering measurements, 
we collected a dataset without pellicle as a reference. We adjusted the exposure time for each measurement in order to obtain the same irradiance value from a clear portion of the sample. This calibration procedure corrects for the different pellicle reflectance values and the variations in the illumination intensity due to the RESCAN optics alignment. Figures 6(a)-6(d) show the reconstructed image magnitude for the reference case, for an MW-CNT pellicle with support mesh, and for two random SW-CNT pellicles with different metal coatings, respectively. From a qualitative inspection, it is evident that the two coated random SW-CNT pellicles have less impact on the image than the aligned MW-CNT pellicle with a support mesh. While Figs. 6(c) and 6(d) do not show any difference from Fig. 6(a), a clear contrast loss and some artifacts can be observed in Fig. 6(b). To evaluate quantitatively, the pellicle's impact on the reconstructed image quality, we measured the image contrast and a created fidelity metric based on the comparison of the images with a reference calculated from the reticle design. The random scattering from the pellicle will contribute to the system flare and reduce the image contrast. To evaluate the contrast of the reconstructed pattern, we separated the bright regions of the image corresponding to the multilayer areas from the dark ones corresponding to the absorber. The selection was carried out using an arbitrary threshold value. We calculated the average and the standard deviation of the intensity in the two regions, and we used the traditional contrast definition

(a)

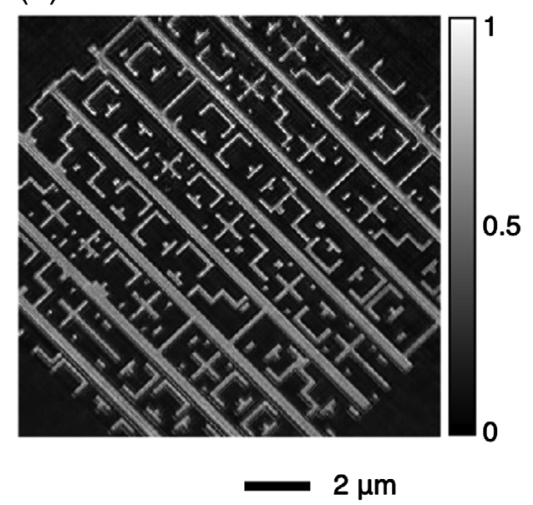

(c)

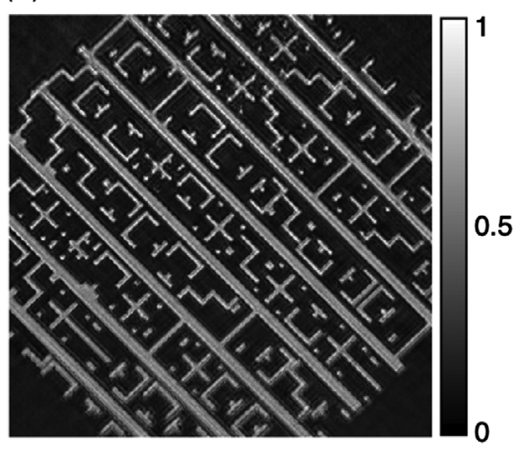

$\mathrm{Cnt}=\frac{\overline{I_{M}}-\overline{I_{m}}}{\overline{I_{M}}+\overline{I_{m}}} \pm 2\left|\frac{\overline{I_{M}} \Delta_{m}-\overline{I_{m}} \Delta_{M}}{\left(\overline{I_{M}}+\overline{I_{m}}\right)^{2}}\right|$,

where $\overline{I_{M}}$ and $\overline{I_{m}}$ are the average intensities of the bright and the dark regions, respectively and $\Delta_{M}$ and $\Delta_{m}$ are the corresponding standard deviations. Since there is a focal gradient in the reconstructed image, this calculation was carried out in a $4 \times 4 \mu \mathrm{m}^{2}$ region centered on the best focus spot.

The values shown in Fig. 7 indicate that if aligned MWCNT pellicles have a stronger scattering contribution, they also have a lower contrast with respect to random coated SW-CNT pellicles. The overall contrast level is remarkably low for all these cases. This is partly due to the fact that this is an average contrast and it is not based on the absolute maximum and minimum values in the images but also on the fact that the absorber of the mask sample under investigation consists of a 140-nm layer of HSQ. The absorption coefficient of HSQ ranges from $4.7 \cdot 10^{-3}$ to $7.4 \cdot 10^{-3} \mathrm{~nm}^{-1}$. Considering a total thickness of $2 \cdot 140 / \cos 6^{\circ} \mathrm{nm}$, the EUV intensity transmitted through the absorber ranges from $12 \%$ to $27 \%$ of the intensity reflected by the bare multilayer, leading to a contrast cap that goes from $73 \%$ to $88 \%$. To evaluate the reconstructed image quality, we defined a fidelity metric based on an ideal reference image. We calculated the reference aerial image by propagating the binary GDS design of the sample through a perfect lens with an aperture equal to the one defined by RESCAN's detector. The reconstructed image and the reference image are aligned using a least

(b)

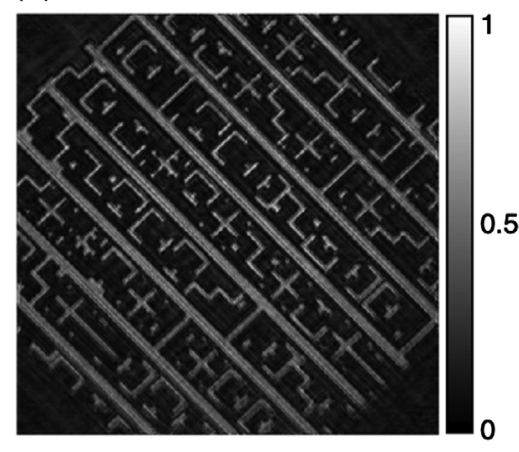

(d)

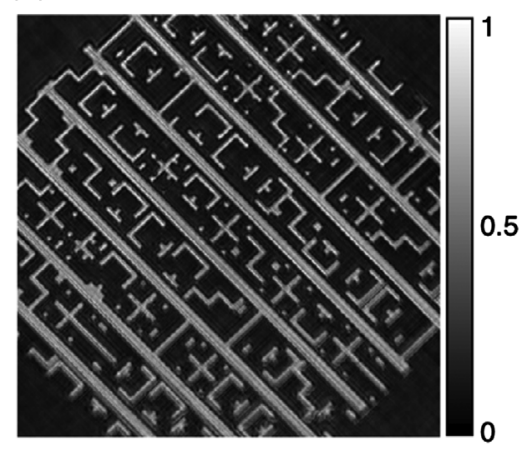

Fig. 6 Through-pellicle image reconstructions. (a) Reference image reconstructed without a pellicle. The CD of the pattern is $200 \mathrm{~nm}$. The sample has a tilt that manifests with a defocus in the lower part of the image. (b) Image reconstruction with an aligned MW-CNT membrane with support mesh. EUV transmittance $\sim 90 \%$. (c) Image reconstruction with a random SW-CNT membrane with a protective metal coating. EUV transmittance $\sim 93 \%$. (D) Image reconstruction with a random SW-CNT membrane with a different protective metal coating. EUV transmittance $\sim 93 \%$. 


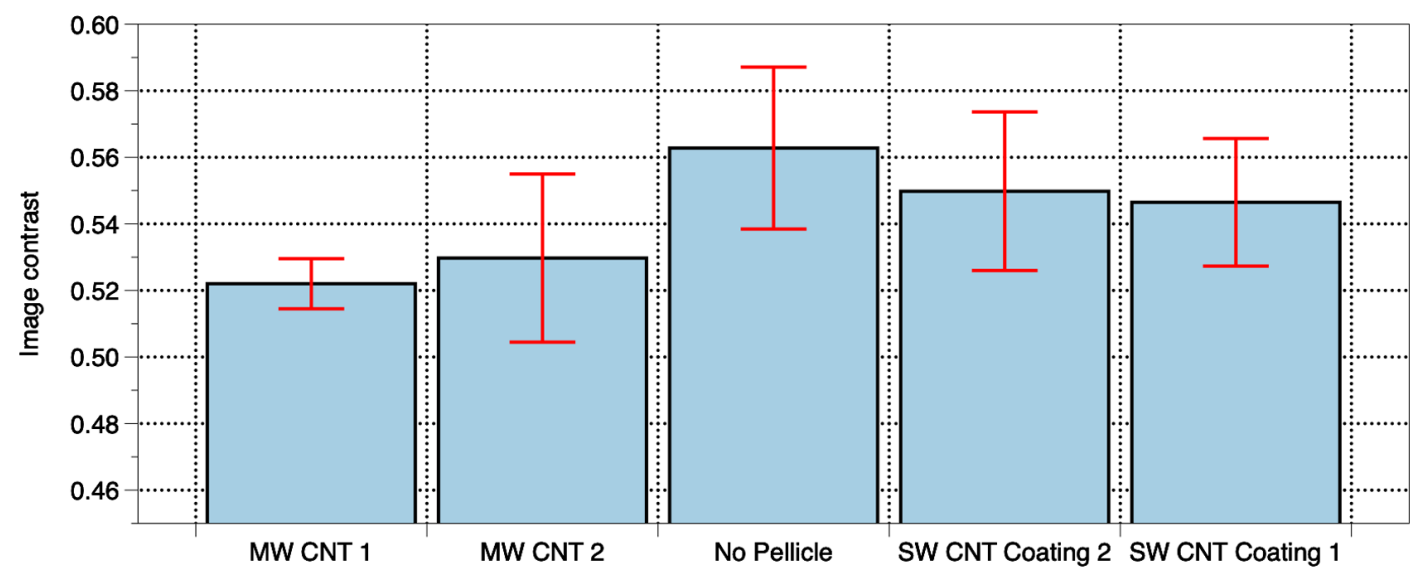

Fig. 7 Average contrast of through pellicle reconstructed images. The contrast is calculated from the average values of the bright and dark regions in the images. The mask sample used is generated by patterning a 140-nm HSQ film on an EUV multilayer designed for maximum reflectivity at 6 deg incidence angle. The membranes exhibiting the strongest scattering show the highest image contrast loss.

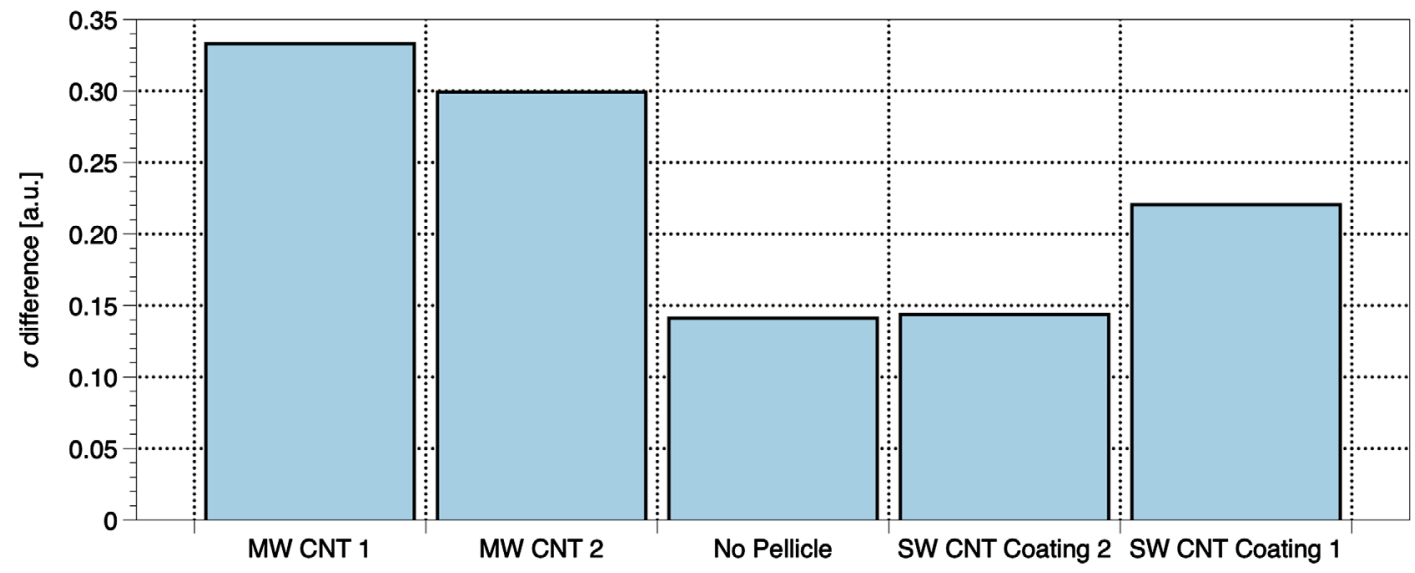

Fig. 8 Reconstructed image fidelity is estimated as the standard deviation of the difference between the reconstructed image and a simulated aerial image calculated from the sample design. The smaller the value of the standard deviation, the higher the reconstructed image fidelity. The reconstruction obtained through the random SW-CNT membrane with metal coating 1 showed little or no difference in image fidelity with respect to the no pellicle case.

square registration algorithm, normalized for best contrast matching and cropped to a $4 \times 4 \mu \mathrm{m}^{2}$ area centered in the best focus spot as done for the contrast evaluation case. The image fidelity is defined as the standard deviation of the difference between the intensity of two images. The lower the standard deviation, the closer the reconstructed image is assumed to be to the ideal case. The results of the image fidelity evaluation are shown in Fig. 8, where we observed that one of the random SW-CNT pellicles with metal coating [corresponding to Fig. 6(c)] exhibits little or no difference from the reference image reconstructed without the pellicle. The image fidelity error in the no-pellicle case depends on the fact that the aerial image that we calculated does not include mask three-dimensional effects or imperfections in the mask pattern.

\section{Conclusions and Outlook}

We used the RESCAN defect inspection platform to investigate the effects of different CNT-based pellicles on the actinic image of EUV mask samples. In particular, we demonstrated a method to measure the pellicle-induced scattering in double-pass mode, we evaluated the contrast loss induced by the pellicle membrane, and we defined an image fidelity metric to compare the effects of the pellicles on the reconstructed image quality. We observed that the metal-coated random SW-CNT membranes show a scattering behavior similar to the bare sample case, in line with the results described by previous studies. ${ }^{9}$ Aligned MW-CNT membranes with large bundles and a support mesh show instead a higher scattering contribution in the spatial frequency region between 0.6 and $2 \mu \mathrm{m}^{-1}$ as shown in Fig. 5. This is due to the directionality of the CNT distribution and suggests that the image of reticle structures in this spatial frequency range may suffer a contrast loss. The image contrast and the fidelity metric we defined showed that metalcoated random SW-CNT pellicles perform better than the aligned MW-CNT ones with a support mesh. In particular, one of the two metal-coated pellicles shows little or no performance difference from the no-pellicle reference case. We also demonstrated that RESCAN can detect pellicle defects that are smaller than $6.5 \mu \mathrm{m}$, which is approximately the smallest dimension that can cause a critical CD error in the 
reticle aerial image. This is an inherent advantage of CDIbased inspection tools that directly record the diffraction patterns of the sample under investigation. Due to this capability, RESCAN can be used not only as an actinic mask inspection platform but also as an efficient tool to inspect the pellicle surface for defects, to characterize its scattering properties, and to evaluate the impact of pellicles on the reticle aerial image contrast and quality.

\section{Acknowledgments}

The authors would like to thank the CNT suppliers, Canatu Oy, and the Nano-Science and Technology Center of Lintec of America Inc., and the PSI ALM technical team, Markus Kropf, Michaela Vockenuber, and José Gabadinho for their contribution to the experiments at the XIL-II beamline. A previous version of this paper was published in the proceedings of the SPIE Photomask Technology and EUV Lithography conference $2018 .^{13}$

\section{References}

1. I. Mochi et al., "Actinic inspection of EUV reticles with arbitrary pattern design," Proc. SPIE 10450, 1045007 (2017).

2. I. Mochi et al., "RESCAN: an actinic lensless microscope for defect inspection of EUV reticles," J. Micro/Nanolithogr. MEMS, MOEMS 16, 041003 (2017).

3. T. Harada et al., "Mask observation results using a coherent extreme ultraviolet scattering microscope at NewSUBARU," J. Vac. Sci. Technol. B 27(6), 3203-3207 (2009).

4. P. Helfenstein et al., "Beam drift and partial probe coherence effects in EUV reflective-mode coherent diffractive imaging," Opt. Express 26, 12242-12256 (2018).

5. C. M. Kewish et al., "Ptychographic characterization of the wavefield in the focus of reflective hard X-ray optics," Ultramicroscopy 110(4), 325-329 (2010)

6. M. Y. Timmermans et al., "CNT EUV pellicle: moving towards a fullsize solution," Proc. SPIE 10451, 10451P (2017).

7. M. Y. Timmermans et al., "Free-standing carbon nanotube films for extreme ultraviolet pellicle application," J. Micro/Nanolithogr. MEMS, and MOEMS 17, 043504 (2018).

8. I. Pollentier et al., "EUV lithography imaging using novel pellicle membranes," Proc. SPIE 9776, 977620 (2016).

9. F. Scholze et al., "EUV optical characterization of alternative membrane materials for EUV pellicles," Proc. SPIE 10451, 104510R (2017).

10. E. Gallagher et al., "CNTs in the context of EUV pellicle history," Proc. SPIE 10583, 105831E (2018).

11. G. Kim et al., "Influence of a wrinkle in terms of critical dimension variation caused by transmission nonuniformity and a particle defect on extreme ultraviolet pellicle," J. Micro/Nanolithogr. MEMS, MOEMS 16, 041008 (2017).

12. H. No et al., "Pattern degradation with larger particles on EUV pellicle," Proc. SPIE 10809, 108091G (2018).

13. I. Mochi et al., "Experimental evaluation of the impact of EUV pellicles on reticle imaging," Proc. SPIE 10810, 108100 Y (2018).

lacopo Mochi is an optical physicist. He started working on EUV mask inspection in 2008 at the Center for X-Ray Optics, where he contributed to the design and development of the SHARP microscope. Later, he joined IMEC as an R\&D engineer studying SRAF solutions to mitigate EUV mask three-dimensional effects. In 2016, he joined Paul Scherrer Institut, where he is currently working on the development of RESCAN, an actinic pattern inspection platform.

Marina Y. Timmermans is a researcher at IMEC, working in the nanoapplications and -materials engineering group. She received her PhD in applied physics at Aalto University, Helsinki, Finland, in 2013, working with carbon nanomaterials synthesis and thin film device applications. After her doctoral research, she has joined IMEC and continued to be actively involved in engineering nanomaterials for various applications with the current focus on EUV pellicle development.

Emily E. Gallagher is a principal member of technical staff at IMEC, focusing on pellicle membrane development, EUV imaging, and photomasks. She received her $\mathrm{PhD}$ in physics studying free-electron lasers before shifting to semiconductors. She worked at IBM, most recently leading the EUV mask development effort, before joining IMEC in 2014.

Marina Mariano is a postdoctoral researcher at IMEC since 2017, in the nanoapplications and -materials engineering group working for the EUV pellicle team. In 2014, she received her PhD in photonics from the Universitat Politècnica de Catalunya (BarcelonaTech) and ICFO-The Institute of Photonic Sciences (Spain). Then, she was YCEI postdoctoral fellow at the Department of Chemical and Environmental Engineering in Yale University, USA. Her current research interests are on electrooptical characterization of innovative materials.

Ivan Pollentier joined IMEC in 1993 after receiving his $\mathrm{PhD}$ in physics engineering from the University of Gent, Belgium, where he became involved in lithography engineering and development of i-line, DUV, and $193 \mathrm{~nm}$ processes toward IMEC's device programs. Since 2007, he has been involved in resist and process related R\&D in the field of EUV lithography.

Rajeev Rajendran graduated from Tata Institute of Fundamental Research, India, in 2012, where he performed experiments aimed at studying the interaction of intense light pulses with matter. After a year as a guest researcher at the University of Maryland, working on laser-based electron acceleration, he went to ETH, Switzerland, where he was involved into the generation of coherent EUV radiation using laser-based high harmonic sources. Currently, he is a postdoctoral scientist at Paul Scherrer Institute and is involved with the RESCAN project.

Patrick Helfenstein is an experimental physicist. He did most of his PhD work at PSI and returned in 2015 for the RESCAN project. He was the first member in the project and has worked on all parts of it. After the initial phase, he concentrated on algorithm development and implementation. He left PSI in 2018 to pursue a career in industrial research.

Sara Fernandez received her master's degree in nanosciences from the University of Paris Sud XI in 2013. Later, she received her PhD from the University of Marseille, in which she used coherent diffraction imaging to characterize single nanostructures. After completing her PhD thesis in 2016, she started applying CDI to EUV photomasks in the context of the RESCAN project. She is currently a postdoctoral scientist at Paul Scherrer Institute.

Dimitrios Kazazis received his $\mathrm{PhD}$ in 2009 from Brown University, USA, working on GeOl tunneling FETs. He did his postdoc until 2014 at CNRS-LPN near Paris, on suspended 2DEGs and quantum Hall effect metrology on graphene. He then joined the Paris Observatory developing THz detectors. Since 2016, he has been working at the Paul Scherrer Institute, Switzerland, on advanced lithography and nanofabrication. He has taught several courses at Brown and at Paris 7 University.

Yasin Ekinci received his $\mathrm{PhD}$ from Max Planck Institute for Dynamics and Self Organization in Göttingen, Germany, in 2003. Since 2009, he has been a senior scientist at Paul Scherrer Institute. He is the head of the Advanced Lithography and Metrology Group in Laboratory for Micro- and Nanotechnology. He works on EUV interference lithography and lensless imaging along with other topics in nanoscience. 